Language and Cognition 10 (2018), 626-640. doi:10.1017/langcog.2018.22

(C) UK Cognitive Linguistics Association, 2019. This is an Open Access article, distributed under the terms of the Creative Commons Attribution licence (http://creativecommons.org/ licenses/by/4.0/), which permits unrestricted re-use, distribution, and reproduction in any medium, provided the original work is properly cited.

\title{
When words burn - language processing differentially modulates pain perception in typical and chronic pain populations
}

\author{
NIKOLA VUKOVIC \\ Department of Psychiatry, University of California San Francisco, \\ United States, and CFIN, Aarhus University, Denmark \\ FRANCESCA FARDO \\ Danish Pain Research Centre, Aarhus, Denmark, and Interacting \\ Minds Centre, Aarhus University, Denmark \\ AND \\ YURY SHTYROV \\ CFIN, Aarhus University, Denmark, and Laboratory of \\ Behavioural Neurodynamics, St. Petersburg University, Russia
}

(Received 10 fuly 2018 - Revised 15 November 2018 - Accepted 29 November 2018 First published online 10 Fanuary 2019)

A B S T RACT

How do we communicate our pain to others? The challenge of conveying such a highly individual experience in words is faced daily by many sufferers of chronic pain and their doctors. Moreover, such linguistic strategies are especially relevant in situations where no obvious reference to physical injuries or tissue damage can be made. Neurolinguistically, this question is directly linked to understanding the brain mechanisms behind the encoding, storage, and comprehension of word meanings. An influential view posits that comprehension involves mentally simulating sensorimotor experiences which words refer to. Here, we test the hypothesis that both pain word comprehension and first-hand experiences of pain rely on a common neural substrate, leading to a prediction that word processing should modulate the perception of noxious stimuli. We used a priming task and asked neurotypical and chronic pain participants to read sentences containing literal or metaphoric pain descriptors, and then rate the intensity of 
thermal pain stimuli. We found that pain language comprehension modulated participants' ratings of pain intensity. Furthermore, this effect depended on linguistic context as well as individual pain history. We discuss our findings within the larger theoretical debate on the nature of semantic representations, and point to their potential relevance for clinical practice.

KEYW ORDS : language, comprehension, pain perception, semantics.

\section{Introduction}

Language forms the foundation of our complex social, economic, and cultural life. Thus, failures to adequately communicate, ranging from basic everyday misunderstandings to clinical language impairments to severe communication deficits, impact not only the quality of life but may have significant societal costs (for example, in the domains of education or healthcare). At the same time, they highlight the need for language science to explain individual differences which shape how word meanings are represented and to clarify why the very same words can lead to cognitive (mis)alignment between people.

One domain often marked by communication problems is the experience of pain. Statistics show that more than $20 \%$ of the population are affected by chronic pain - a condition which has negative impacts on people's comfort, career, and general ability to perform functional activities in their daily life (Van Hecke, Torrance, \& Smith, 2013). While most individuals experience pain in life, it is important to appreciate the highly individualised way in which pain presents itself and the inherent difficulties of communicating such personal experience. This is especially true with chronic pain which cannot always be explained in terms of direct injury or tissue damage though patients often describe it as such through the use of literal and metaphoric pain descriptions (Lascaratou, 2007). Existing reports from a 1994 US Harris poll and the UK's Patient and Client Council (PCC) highlight very frequent communication problems between patients and clinical practitioners (see Katz, 1998; PCC, 2014). In both clinical and everyday settings, the question of how pain can be communicated (or miscommunicated) is directly linked to theories of how we encode, store, and comprehend semantic content. What mechanisms do we use to understand and represent words referring to painful experiences, and do these representations differ between individuals?

Recent work in psychology and neuroscience suggests that word meaning is not abstract, but is constructed during comprehension using basic sensorimotor mechanisms (Anderson, 2010; Binder \& Desai, 2011; Kiefer \& Barsalou, 2013; Pulvermüller, 2018). For example, studies show 
that understanding visual, auditory, or motor-related words is achieved by mentally simulating relevant perceptual or action properties in visual, auditory, and motor brain areas, respectively (Halpern, Zatorre, Bouffard, \& Johnson, 2004; Hauk, Johnsrude, \& Pulvermüller, 2004; Horton \& Rapp, 2003; Shtyrov, Butorina, Nikolaeva, \& Stroganova, 2014; Vukovic, Feurra, Shpektor, Myachykov, \& Shtyrov, 2016; Vukovic \& Shtyrov, 2014; Vukovic \& Williams, 2014; Zwaan \& Pecher, 2012). Second, the brain uses an individual's unique collection of embodied conceptual knowledge and experiences to make sense of incoming speech. Accordingly, results from numerous studies show that mental simulation is not an all-or-nothing process but is shaped by individual cognitive differences and experience, and causally relies on sensorimotor brain structures (Beilock, Lyons, Mattarella-Micke, Nusbaum, \& Small, 2008; Tschentscher, Hauk, Fischer, \& Pulvermüller, 2012; Vukovic \& Williams, 2015; Wassenburg \& Zwaan, 2010; Yee, 2017). Very little work, however, has focused on studying the relationship between language and other less dominant senses, such as nociception - the system underlying the detection of potentially harmful stimuli. Indeed, it has been questioned whether an account of language comprehension based on sensorimotor mental simulation could satisfactorily explain the encoding and storage of words related to experiences arising from internal inputs or less dominant modalities. For example, recent experiments which find that words related to odour do not seem to involve simulation (Speed \& Majid, 2018) - unlike those related to vision - highlight the need to study language comprehension in relation to perceptual modalities which are less dominant, or not directly accessible by other individuals.

The 'mental simulation' account of word processing would predict that understanding pain language and actual pain perception share a common neural substrate. If this is true, then mere comprehension of pain-related language should engage representations on which pain perception operates, and thus the two domains should interact. However, very little research exists probing the relationship of language and nociception / pain perception. One relevant example is a study by Richter, Eck, Straube, Miltner, and Weiss (2010), which showed that deliberate pain imagery cued by pain words led to pain-associated brain activity in normal individuals. Another study by Reuter, Werning, Kuchinke, and Cosentino (2017) found that people with greater self-reported pain sensitivity have stronger word-pain associations. To our knowledge, no study to date has addressed the matter of storage and implicit processing of pain semantics in neurotypical and chronic pain populations, and how linguistic context may modulate pain perception.

The aim of our study was to test the influence of language comprehension on pain perception, in the context of semantic models based on mental simulation (Barsalou, Santos, Simmons, \& Wilson, 2008). Specifically, we 
hypothesised that comprehension of verbal pain descriptors involves pain simulations, which influence (i.e., prime) the perception of incoming noxious stimuli. Second, we hypothesised a modulatory role of linguistic context, which was operationalised by presenting pain descriptors either in literal sentences, with direct reference to actual pain episodes (e.g., 'burning' pain after touching the stove), or in metaphoric sentences, in which the same pain descriptors were used to refer to abstract events (e.g., 'burning' issue under discussion). We expected literal pain sentences to increase the perceived intensity of pain more than metaphoric ones, due to the more abstract linkage between the two domains in the case of figurative language (Pecher, Boot, \& Van Dantzig, 2011; Yang \& Shu, 2016). Third, we hypothesised that individual differences play a role in pain-language comprehension, and that personal pain history will modulate the effects of language on perception. Specifically, we expected pain-language to exert an hyperalgesic effect (i.e., increased evaluation of pain). To assess this hypothesis, we compared responses of neurotypical individuals to those of patients suffering from chronic pain due to migraine. We expected stronger priming effects of language on pain perception in patients with chronic migraine, given that their experiences predispose them to have stronger associative connections between language and pain-related networks. Understanding whether and how language modulates pain perception in different contexts and populations would offer key insights into how semantic content is represented, and the cognitive factors which shape pain perception and regulation.

\section{Methods}

\subsection{PART I C I PAN T S}

We recruited 40 right-handed (Oldfield, 1971) participants: 20 chronic headache sufferers (Mean age $=23.2, \mathrm{SD}=3.23$ ) and 20 neurotypical controls (Mean age $=23.0, \mathrm{SD}=1.75)$. Due to the fact that migraine prevalence disproportionately affects women (Peterlin, Gupta, Ward, \& MacGregor, 2011; Waters \& O’Connor, 1975), we only recruited female participants in this study. Data from one chronic pain participant was lost due to technical error, thus subsequent analyses are based on the remaining 19. On average, these participants first started experiencing significant head pain at the age of $14.23(\mathrm{SD}=5.43)$, and reported suffering 9.06 individual headache attacks per month $(\mathrm{SD}=5.45)$, with an average pain intensity of $6.73(\mathrm{SD}=1.62$; where 0 is 'no pain' and 10 is 'worst imaginable pain'). Both the control and chronic pain participants were native Danish speakers, with normal or corrected-to-normal vision, had no history of language disorders, and were otherwise healthy. Before taking part in the experiment, participants provided written informed consent and were monetarily compensated for their time. 
All study protocols were conducted in accordance with the Declaration of Helsinki and were approved by the Research Ethics Committee of Central Jutland Region in Denmark.

\subsection{LANGUAGE STIMULI}

To investigate the cognitive processing of pain-language and its interplay with pain perception, participants completed a sentence comprehension and pain rating task. In this task, participants read a series of Danish sentences containing pain descriptors which were used in either literal (e.g., [translation from Danish] "Anna spilled acid on her hands - it felt burning"; "Vibeke suffered from severe arthritis - any movement felt piercing”) or metaphoric contexts ("Erik's team lost 0 to 4 - the defeat was burning"; "Jen's girlfriend saw him with another woman - her stare was piercing”). Because we were specifically interested in the interaction of pain perception and pain-word meaning, as opposed to general reading processes, we also included a set of non-pain related sentences. These sentences served as a semantic baseline and ended in neutral adjectives ("Laura repainted the wall - the change was cosmetic"; "Emil tried to assemble the IKEA cabinet - the manual was technical”). Forty trials of each sentence type (literal pain, metaphoric pain, neutral) were presented, for a total of 120 trials.

\subsection{TASK PROCEDURE}

On each trial participants were shown a fixation cross in the centre of a screen for $500 \mathrm{~ms}$, followed by a sentence presented word-by-word. Depending on word length (which was balanced across conditions), words appeared on the screen for between 500 and $800 \mathrm{~ms}$ (sampled in steps of $100 \mathrm{~ms}$ ). The final word in the sentence was always the pain or neutral descriptor which was presented for $1000 \mathrm{~ms}$. At sentence offset, another fixation cross was presented for $500 \mathrm{~ms}$, following which participants received a single heat pain stimulus on the forearm of their non-dominant (i.e., left) hand, while the dominant hand was used to provide responses. To reduce pain onset predictability, the thermal stimulus was delivered randomly within a 2 -second time-window. Participants then had up to 5 seconds to rate the subjectively perceived intensity of the sensation (see pain stimulation details below), using a visual numerical scale from 0 ('no pain') to 10 ('worst imaginable pain'), in steps of 0.5 . To ensure they read all the sentences carefully, at the end of $30 \%$ of trials participants had to answer a yes/no comprehension question. In total, each participant completed 120 trials - of these 40 contained neutral, 40 literal, and 40 metaphoric pain sentences (for a list of all sentences used, see $<$ https:// osf.io/a23jc/>). 


\subsection{PAIN STIMULATION}

The thermal stimulation was delivered using a Contact Heat Evoked Potential Stimulator (CHEPS, Medoc Ltd., Ramat Yishai, Israel) with a circular contact area of 573 square $\mathrm{mm}$, applied to the volar surface of the left forearm. The right (i.e., dominant) arm was used for providing responses and pain ratings. Stimuli consisted of two possible heat pulses at low and high intensity, from a baseline temperature of $32^{\circ} \mathrm{C}$. Stimulus temperatures were calibrated for each participant and corresponded to ratings of 2 (low intensity) and 6 (high intensity) on a scale from 0 ('no pain') to 10 ('worst imaginable pain'). For all stimuli, the heating rate was $70^{\circ} \mathrm{C} / \mathrm{s}$ with a return rate of $40^{\circ} \mathrm{C} / \mathrm{s}$, producing a baseline-to-peak heat pulse in around $400 \mathrm{~ms}$. Participants felt pain mainly during the peak portion. For each language category (neutral, literal, metaphorical pain sentences), we delivered an equal number of low or high painful stimulation. Further, sentence stimuli were delivered in randomised order and each sentence was repeated once for both stimulation intensities.

\subsection{STATISTICAL ANALYSIS}

The pain modulation effect of literal and metaphoric sentences was expressed as percent change in pain ratings relative to the neutral sentence baseline (which contained no pain-related words). These values were entered as a dependent variable in a three-way repeated measures ANOVA with the factors Sentence Type (literal vs. metaphoric sentence) and Pain Intensity (low vs. high), and the between-participant factor of Group (chronic pain vs. control participants). Post-hoc pairwise comparisons were conducted using planned $t$-tests. In addition, we performed the same analysis on mean RTs (reaction times) as the dependent variable in order to explore any response speed differences between conditions. Analyses were conducted using the JASP statistical software (JASP Team, 2018). Data and analysis materials for this study are freely available at the Open Science Framework (<https://osf.io/a23jc/ $>$ ).

\section{Results}

\subsection{PAIN CALIBRATION}

Average temperature and standard deviation of the thermal pain stimulation were $43.33 \pm 1.30^{\circ} \mathrm{C}$ for the low pain intensity, and $49.72 \pm 0.92^{\circ} \mathrm{C}$ for the high pain intensity. No significant difference in the average pain intensity was found between chronic pain patients and controls, for either the low$(t(37)=1.59, p=.12$, Cohen's $d=0.51)$ or the high-intensity stimuli $(t(37)=1.66, p=.10$, Cohen's $d=0.53)$. This means that the objective stimulus temperatures were comparable across the two groups of participants. 


\subsection{PAIN RATING TASK}

Figure 1 shows the effects of language comprehension on pain perception in chronic pain patients and neurotypical controls. The dependent variable corresponds to the pain modulation effect of literal and metaphoric sentences relative to neutral sentences, expressed as a percentage change. We found both a Sentence Type main effect $\left(F(1,37)=17.01, p<.001\right.$, partial $\left.\eta^{2}=0.31\right)$, indicating that literal sentences modulated subsequent pain ratings more than metaphoric sentences did, as well as a Pain Intensity main effect $(F(1,37)$ $=26.61, p<.001$, partial $\left.\eta^{2}=0.42\right)-$ meaning that larger changes in pain ratings were associated with low-intensity relative to high-intensity pain stimulation. This suggests an allodynic effect (i.e., pain perception from lowintensity stimuli), where the perception of weaker painful stimuli is selectively enhanced. Further, there were significant interactions between Sentence Type and Pain Intensity $(F(1,37)=10.08, p=.003,=0.21)$ and between Sentence Type and Group $\left(F(1,37)=5.64, p=.023\right.$, partial $\left.\eta^{2}=0.13\right)$. Specifically, literal sentences enhanced pain to a larger extent relative to metaphoric sentences in cases of low-intensity pain stimulation. The allodynic effect was thus larger when participants were primed with literal pain-related sentences. Conversely, literal and metaphoric sentences had little influence on pain perception of high-intensity stimuli. Crucially, pain modulation exerted by literal vs. metaphoric sentences was higher in chronic pain patients compared to controls. No other significant effects emerged (Group main effect: $F(1,37)=0.38, p=.54$, partial $\eta^{2}=0.01$; Pain Intensity*Group interaction: $F(1,37)=0.29, p=.59$, partial $\eta^{2}=0.008$; Sentence*Pain Intensity*Group interaction: $F(1,37)=0.87, p=.36$, partial $\left.\eta^{2}=0.02\right)$. The same ANOVA conducted on mean RTs as a dependent variable returned no significant main effects or interactions (all $p \mathrm{~s}>.05$ ), indicating that participants responded similarly quickly in all conditions.

\section{Discussion}

The present study sought to address the issue of whether and how symbolic linguistic representations interact with perceptual mechanisms during language comprehension (Binder \& Desai, 2011; Horchak, Giger, Cabral, \& Pochwatko, 2014; Meteyard, Cuadrado, Bahrami, \& Vigliocco, 2012; Pulvermüller, 2013) in the context of pain experience (Schott, 2004; Semino, 2010; Van Wijk \& Hoogstraten, 2004). First, we hypothesised that understanding the meaning of pain-related words (e.g., 'burning', 'piercing') is achieved by an automatic simulation of pain, possibly involving neural mechanisms that also participate in first-hand pain perception. On this view, we expected language to modulate the perception of pain evoked by noxious stimulation. Second, in agreement with previous language comprehension 


\section{A Experimental Procedure Trial timeline and thermal stimulation}

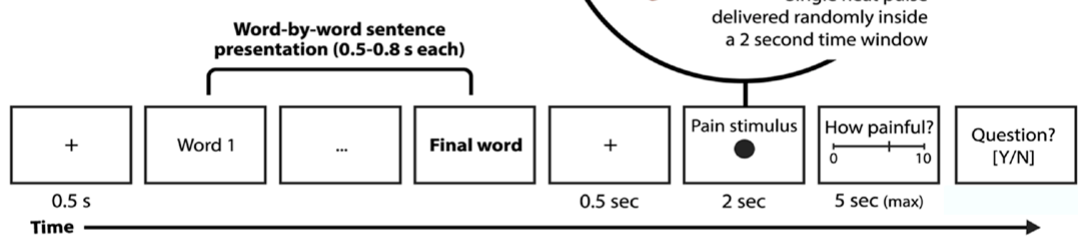

B

Main Effect: Intensity

Main Effect: Sentence

Sentence * Intensity

Sentence * Group
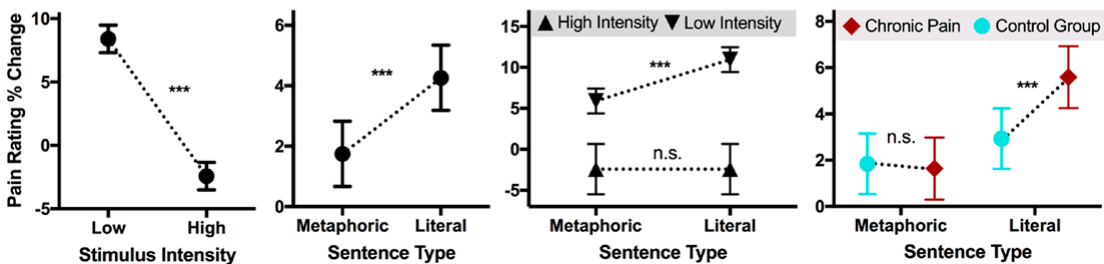

Fig. 1. Experimental procedure and results. A. On each trial, normal and chronic pain participants viewed neutral, literal, and metaphoric pain sentences. Sentences were displayed word by word, after which one thermal pain stimulus was delivered to their left forearm. Participants rated the perceived intensity of the noxious stimulus by clicking with their right hand on a visual numerical scale. On $30 \%$ of trials, a comprehension question about the previously read sentence was presented. B. Results show the percentage change pain modulation effect of literal and metaphoric pain sentences relative to the neutral sentence baseline $( \pm \mathrm{SEM})$. Left to right, we see that our procedure was successful in establishing two distinct pain categories (low and high), and that the perceived pain intensity was overall modulated by sentential context (greater priming in literal context). Moreover, we found that this modulatory effect was specific to lower-intensity stimuli, and that literal pain sentences had the strongest hyperalgesic influence in chronic pain patients.

literature (Cosentino, Baggio, Kontinen, \& Werning, 2017; Just, Wang, \& Cherkassky, 2017; Knoeferle, Urbach, \& Kutas, 2011; Maguire, Frith, \& Morris, 1999), we expected the degree of pain modulation to be influenced by the semantic context. We manipulated such context by using sentences describing pain experiences either in a metaphoric or in a literal fashion. The pain-related sentences were compared to baseline sentences containing only pain-unrelated words. Finally, given that pain is experienced in highly individualised ways in relation to quality, intensity, and history (e.g., in chronic pain), we hypothesised that prior history of chronic pain may influence the interplay between language comprehension and pain perception. Specifically, we expected the magnitude of linguistic priming on pain 
perception to be higher in chronic pain sufferers, due to stronger associative connections between language and pain networks, compared to healthy controls. Our results confirm all three hypotheses, and show that pain sentences modulate actual experiences of pain, especially when literal expressions are used, and that such priming is more pronounced in individuals with a history of chronic pain.

The first novel finding of our study is that language comprehension modulates the perceived intensity of pain stimuli, and that the extent of this language-driven modulation is dependent on the context. The very same pain descriptors influenced subsequent pain ratings differently, based on whether they were embedded in metaphoric or literal sentences. We found that both types of sentences increased pain intensity ratings relative to a neutral semantic baseline; however, sentences describing literal pain events did so to a greater extent. This finding supports the interpretation that language comprehension involves mentally simulating word and sentence meaning in a flexible manner, possibly using the same resources involved in the representation of pain itself. These findings also speak to polysemy accounts of metaphor comprehension, which stress that novel metaphors (of the kind used here) entail a representational shift with respect to the source semantic domain (Bowdle \& Gentner, 2005; Klepousniotou \& Baum, 2005; Lehrer, 1990). The meaning of figurative expressions is therefore more flexible than the meaning of literal ones, and is shaped by context and level of conventionality in both the timecourse of activation of word senses, and the frequency/dominance of these senses.

We further show that the priming effect of language varies with prior pain history. In the control group, literal pain sentences led to only marginally larger pain ratings compared to metaphoric sentences, suggesting that these participants represented them similarly with respect to pain content. However, the group of chronic pain sufferers showed a much larger difference between linguistic contexts. Whereas metaphoric pain language elicited similar responses in both groups of participants, chronic migraine patients rated noxious stimuli as more painful when they were primed with a literal pain sentence. This finding suggests a role of personal experience on the way we represent and understand the meaning of words related to pain. This is in agreement with linguistic and semantic theories suggesting a role of bodily experience in language comprehension (Barsalou, 1999; Borghi, Flumini, Cimatti, Marocco, \& Scorolli, 2011; Meltzoff, 1990; Pexman, 2017). This finding complements previous studies in the literature that have focused on perceptual domains that are highly shared among individuals, such as vision or action. By zooming in on an internal domain of experience which may have much less overlap between people, we were able to demonstrate significant differences between groups in the degree to which language comprehension 
can potentiate the perception of pain. This finding is consistent with the results of a questionnaire study conducted by Reuter et al. (2017), which found that pain-sensitive people associate words more strongly with pain than less sensitive individuals.

The present study also indicates that the influence of language on pain perception might depend on the intensity of painful stimulation, as we observed modulation of pain ratings for low- but not high-intensity stimuli. It is possible that subtle linguistic manipulations of the type used here are overshadowed by high-intensity noxious stimulation, which is comparatively more intense and might obscure any linguistic effects (i.e., ceiling effect). Arguably, linguistic modulations of high-intensity stimulation might be evident when using other protocols - for example, as part of longer-term therapeutic interventions, or tasks which involve more deliberate semantic processing. Indeed, previous studies demonstrated modulation of highintensity stimuli when participants were instructed to use hypoalgesic (i.e., pain-reducing) or hyperalgesic (pain-increasing) mental imagery prior to pain stimulation (Fardo, Allen, Jegindø, Angrilli, \& Roepstorff, 2015); this was found when attention was manipulated (Fardo, Auksztulewicz, Allen, Dietz, Roepstorff, \& Friston, 2017) and when participants explicitly learnt about the probability of painful occurrences via cue-conditioning paradigms (Egorova, Park, \& Kong, 2017; Jepma \& Wager, 2015; Keltner, 2006). In an fMRI study, instructing people to explicitly imagine pain when cued by painrelated words led to increased activation in areas of the cortical network known to mediate the perception, localisation, and encoding of painful stimuli (Richter et al., 2010) - though this study looked at normal participants only, and did not measure pain priming or perception itself. However, the process of generating conscious mental imagery is considered to be related but distinct from automatic and largely implicit language comprehension processes (e.g., Barsalou, 1999; Gold, Balota, Jones, Powell, Smith, \& Andersen, 2006; Graves, Binder, Desai, Conant, \& Seidenberg, 2010; Neely, Keefe, \& Ross, 1989; Salles, Holderbaum, Parente, Mansur, \& Ansaldo, 2012). In sum, our study demonstrates that a subtle and incidental semantic processing task can modulate the perception of low-intensity stimuli, but possibly also more intense painful stimulation under other language circumstances or tasks, which remains to be elucidated in more detail in future studies.

It is worth noting that, while the pain modulation effect of sentences (and especially the observed group differences) is consistent with and predicted by semantic theories of word representation, other alternative accounts could be proposed. One such explanation could be based on attentional bias, whereby highly pain-sensitive individuals are prone to attend more to pain-related stimuli because of additional experience (Baum, Huber, Schneider, \& Lautenbacher, 2011). Some evidence for this claim 
comes from a study of Pearce and Morley (1989), who found that chronic pain patients were slower than controls to name the colour of a word in a Stroop task if the word was pain-related. However, in the present study we find no support for the hypothesis that chronic participants attended preferentially to pain language, since there were no reaction time differences between conditions or between the two groups. Further, chronic pain individuals and controls were well matched in terms of pain sensitivity, given that their individually calibrated pain stimulation thresholds did not differ. Thus, given the similar reaction times in response to pain-related words and similar pain sensitivity, we believe the attentional bias explanation is unlikely. Rather, an appeal to semantic processes related to meaning representation and comprehension provide a more parsimonious explanation for the present effect of linguistic modulation of pain perception.

Taken together, our results speak to the integral role that perceptual mechanisms play in the processing of language. They demonstrate that semantic representations are tied to processes which mediate our perception of both the world and ourselves (Anderson, 2010; Barsalou, 2008), while highlighting the important role of linguistic context. Thus, they are in line with flexible accounts of word comprehension which stress that sentence context drives how lexical and semantic information is accessed (Connell \& Lynott, 2014; Marslen-Wilson \& Tyler, 1980). While we found that both metaphoric and literal uses of pain language prime the perception of noxious stimuli, relative to a neutral baseline, the magnitude of this effect seems inversely related to the degree of abstractness evoked by the sentence. Moreover, our findings raise the need for researchers to consider factors other than just linguistic features of words which people are asked to process, such as the individual differences in experience between people. Indeed, we found larger priming effects for literal language in sufferers of chronic pain, suggesting that chronic pain might implicate stronger associative connections between language and pain networks. This insight may prove particularly relevant in the clinical context where significant communication problems exist between patients and medical practitioners (Katz, 1998; PCC, 2014). Given that chronic pain is a major burden affecting around $20 \%$ of the population, and that painful symptoms are the most common reason for which individuals seek medical attention, incorporating these insights from language science into practical improvements in doctor-patient communication in relation to pain diagnosis and treatment may be an exciting prospect.

\section{Conclusion}

While there is mounting evidence for the view that language comprehension is mediated by the brain's sensory and motor systems, it remains unclear 
whether this account can adequately explain the meanings of words related to less dominant modalities, and what the role of experience is in shaping these representations. By investigating the relationship between online language processing and pain perception in populations with different prior history of chronic pain, we were able to show that the comprehension of pain words may be mediated by mechanisms engaged in experiencing pain itself. Moreover, we find that language primes pain perception in a way that is sensitive to sentence context and individual pain experience. Thus, our findings enrich existing debates in semantic theory as well as highlight their potential relevance to clinical practice and the improvement of doctor-patient communication.

\section{Data availability}

Data and materials for this study are freely available at the Open Science Framework (<https://osf.io/a23jc/>).

\section{Acknowledgements}

This work was supported by the Interacting Minds Centre at Aarhus University (project grant 2017-26111, NV), Lundbeck Foundation (R1402013-12951, project 15480, YS), Danish Council for Independent Research (6110-00643B; FF), and RF government (grant contract 14.W03.31.0010, YS). We would like to thank Andreas Højlund-Nielsen and Stine Sørensen for their help in stimuli selection, as well as Camilla Andersen and Caroline Børsting for helping with data collection and participant recruitment.

\section{REFEREN CES}

Anderson, M. L. (2010). Neural reuse: a fundamental organizational principle of the brain. Behavioral and Brain Sciences, 33(4), 245-266.

Barsalou, L. W. (1999). Perceptual symbol systems. Behavioral and Brain Sciences, 22(4), $577-609$

Barsalou, L. W. (2008). Grounded cognition. Annual Review of Psychology, 59, 617-645.

Barsalou, L. W., Santos, A., Simmons, W. K., \& Wilson, C. D. (2008). Language and simulation in conceptual processing. In M. de Vega, A. M. Glenberg, \& A. C. Graesser (Eds.), Symbols and embodiment: debates on meaning and cognition (pp. 245-284). Oxford: Oxford University Press.

Baum, C., Huber, C., Schneider, R., \& Lautenbacher, S. (2011). Prediction of experimental pain sensitivity by attention to pain-related stimuli in healthy individuals. Perceptual and Motor Skills, 112(3), 926-946.

Beilock, S. L., Lyons, I. M., Mattarella-Micke, A., Nusbaum, H. C., \& Small, S. L. (2008). Sports experience changes the neural processing of action language. Proceedings of the National Academy of Sciences of the United States of America, 105(36), 13269-13273.

Binder, J. R., \& Desai, R. H. (2011). The neurobiology of semantic memory. Trends in Cognitive Sciences, 15(11), 527-536. 
Borghi, A. M., Flumini, A., Cimatti, F., Marocco, D., \& Scorolli, C. (2011). Manipulating objects and telling words: a study on concrete and abstract words acquisition. Frontiers in Psychology, 2, 1-14. Online <https://doi.org/10.3389/fpsyg.2011.00015>.

Bowdle, B. F., \& Gentner, D. (2005). The career of metaphor. Psychological Review, 112(1), 193-216.

Connell, L., \& Lynott, D. (2014). Principles of representation: why you can't represent the same concept twice. Topics in Cognitive Science, 1-17. Online <https://doi.org/10.1111/ tops.12097>.

Cosentino, E., Baggio, G., Kontinen, J., \& Werning, M. (2017). The time-course of sentence meaning composition: N400 effects of the interaction between context-induced and lexically stored affordances. Frontiers in Psychology, 8, 1-17. Online <https://doi.org/10.3389/ fpsyg.2017.00813>.

Egorova, N., Park, J., \& Kong, J. (2017). In the face of pain: the choice of visual cues in pain conditioning matters. European Fournal of Pain (United Kingdom), 21(7), 1243-1251.

Fardo, F., Allen, M., Jegindø, E. M. E., Angrilli, A., \& Roepstorff, A. (2015). Neurocognitive evidence for mental imagery-driven hypoalgesic and hyperalgesic pain regulation. NeuroImage, 120, 350-361.

Fardo, F., Auksztulewicz, R., Allen, M., Dietz, M. J., Roepstorff, A., \& Friston, K. J. (2017). Expectation violation and attention to pain jointly modulate neural gain in somatosensory cortex. NeuroImage, 153, 109-121.

Gold, B. T., Balota, D. A., Jones, S. J., Powell, D. K., Smith, C. D., \& Andersen, A. H. (2006). Dissociation of automatic and strategic lexical-semantics: functional magnetic resonance imaging evidence for differing roles of multiple frontotemporal regions. Fournal of Neuroscience, 26(24), 6523-6532.

Graves, W. W., Binder, J. R., Desai, R. H., Conant, L. L., \& Seidenberg, M. S. (2010). Neural correlates of implicit and explicit combinatorial semantic processing. NeuroImage, 53(2), $638-646$.

Halpern, A. R., Zatorre, R. J., Bouffard, M., \& Johnson, J. A. (2004). Behavioral and neural correlates of perceived and imagined musical timbre. Neuropsychologia, 42, 1281-1292.

Hauk, O., Johnsrude, I., \& Pulvermüller, F. (2004). Somatotopic representation of action words in human motor and premotor cortex. Neuron, 41(2), 301-307.

Horchak, O. V., Giger, J.-C., Cabral, M., \& Pochwatko, G. (2014). From demonstration to theory in embodied language comprehension: a review. Cognitive Systems Research, 29/30, 66-85.

Horton, W. S., \& Rapp, D. N. (2003). Out of sight, out of mind: occlusion and the accessibility of information in narrative comprehension. Psychonomic Bulletin $\xi^{\circ}$ Review, 10, 104-110.

JASP Team (2018). JASP (Version 0.8.6) [Computer software].

Jepma, M., \& Wager, T. D. (2015). Conceptual conditioning: mechanisms mediating conditioning effects on pain. Psychological Science, 26(11), 1728-1739.

Just, M. A., Wang, J., \& Cherkassky, V. L. (2017). Neural representations of the concepts in simple sentences: concept activation prediction and context effects. NeuroImage, 157, 511-520.

Katz, W. A. (1998). The needs of a patient in pain. American fournal of Medicine, 105(1 B), 2S-7S.

Keltner, J. R. (2006). Isolating the modulatory effect of expectation on pain transmission: a functional magnetic resonance imaging study. Fournal of Neuroscience, 26(16), 4437-4443.

Kiefer, M., \& Barsalou, L. W. (2013). Grounding the human conceptual system in perception, action, and internal states. In W. Prinz, M. Beisert, \& A. Herwig (Eds.), Action science: foundations of an emerging discipline (pp. 381-407). Cambridge, MA: MIT Press.

Klepousniotou, E., \& Baum, S. R. (2005). Processing homonymy and polysemy: effects of sentential context and time-course following unilateral brain damage. Brain and Language, 95(3), 365-382.

Knoeferle, P., Urbach, T. P., \& Kutas, M. (2011). Comprehending how visual context influences incremental sentence processing: insights from ERPs and picture-sentence verification. Psychophysiology, 48(4), 495-506. 


\section{WHEN WORDS BURN}

Lascaratou, C. (2007). The language of pain: expression or description? Amsterdam: John Benjamins.

Lehrer, A. (1990). Polysemy, conventionality, and the structure of the lexicon. Cognitive Linguistics. Online <https://doi.org/10.1515/cogl.1990.1.2.207>.

Maguire, E. A., Frith, C. D., \& Morris, R. G. M. (1999). The functional neuroanatomy of comprehension and memory: the importance of prior knowledge. Brain, 122(10), 1839-1850.

Marslen-Wilson, W., \& Tyler, L. K. (1980). The temporal structure of spoken language understanding. Cognition, 8(1), 1-71.

Meltzoff, A. N. (1990). Towards a developmental cognitive science: the implications of crossmodal matching and imitation for the development of representation and memory in infancy. Annals of the New York Academy of Sciences, 608(1), 1-37.

Meteyard, L., Cuadrado, S. R., Bahrami, B., \& Vigliocco, G. (2012). Coming of age: a review of embodiment and the neuroscience of semantics. Cortex, 48(7), 788-804.

Neely, J. H., Keefe, D. E., \& Ross, K. L. (1989). Semantic priming in the lexical decision task: roles of prospective prime-generated expectancies and retrospective semantic matching. Fournal of Experimental Psychology: Learning, Memory, and Cognition, 15(6), 1003-1019.

Oldfield, R. C. (1971). The assessment and analysis of handedness: the Edinburgh inventory. Neuropsychologia, 9, 97-113.

PCC (Patient and Client Council) (2014). The painful truth. Online: <http://www. patientclientcouncil.hscni.net/publications/index/reports/date/2014>.

Pearce, J., \& Morley, S. (1989). An experimental investigation of the construct validity of the McGill Pain Questionnaire. Pain, 39, 115-121.

Pecher, D., Boot, I., \& Van Dantzig, S. (2011). Abstract concepts: sensory-motor grounding, metaphors, and beyond. Psychology of Learning and Motivation, 54, 1-44.

Peterlin, B. L., Gupta, S., Ward, T. N., \& MacGregor, A. (2011). Sex matters: evaluating sex and gender in migraine and headache research. Headache: The fournal of Head and Face Pain, 51(6), 839-842.

Pexman, P. M. (2017). The role of embodiment in conceptual development. Language, Cognition and Neuroscience. Online <https://doi.org/10.1080/23273798.2017.1303522>.

Pulvermüller, F. (2013). How neurons make meaning: brain mechanisms for embodied and abstract-symbolic semantics. Trends in Cognitive Sciences, 17(9), 458-470.

Pulvermüller, F. (2018). Neural reuse of action perception circuits for language, concepts and communication. Progress in Neurobiology, 160, 1-44.

Reuter, K., Werning, M., Kuchinke, L., \& Cosentino, E. (2017). Reading words hurts: the impact of pain sensitivity on people's ratings of pain-related words. Language and Cognition, 9(3), 533-567.

Richter, M., Eck, J., Straube, T., Miltner, W. H. R., \& Weiss, T. (2010). Do words hurt? Brain activation during the processing of pain-related words. Pain, 148(2), 198-205.

Salles, J. F. de, Holderbaum, C. S., Parente, M. A. M. P., Mansur, L. L., \& Ansaldo, A. I. (2012). Lexical-semantic processing in the semantic priming paradigm in aphasic patients. Arquivos de Neuro-Psiquiatria, 70(9), 718-726.

Schott, D. G. (2004). Communicating the experience of pain: the role of analogy. Pain, 108(3), 209-212.

Semino, E. (2010). Descriptions of pain, metaphor, and embodied simulation. Metaphor and Symbol, 25(4), 205-226.

Shtyrov, Y., Butorina, A., Nikolaeva, A., \& Stroganova, T. (2014). Automatic ultrarapid activation and inhibition of cortical motor systems in spoken word comprehension. Proceedings of the National Academy of Sciences of the United States of America, 111(18) E1918-E1923. Online <https://doi.org/10.1073/pnas.1323158111>.

Speed, L. J., \& Majid, A. (2018). An exception to mental simulation: no evidence for embodied odor language. Cognitive Science, 42(4), 1146-1178.

Tschentscher, N., Hauk, O., Fischer, M. H., \& Pulvermüller, F. (2012). You can count on the motor cortex: finger counting habits modulate motor cortex activation evoked by numbers. NeuroImage, 59(4), 3139-3148. 
Van Hecke, O., Torrance, N., \& Smith, B. H. (2013). Chronic pain epidemiology and its clinical relevance. British Fournal of Anaesthesia, 111(1), 13-18.

Van Wijk, A. J., \& Hoogstraten, J. (2004). Paired comparisons of sensory pain adjectives. European Fournal of Pain, 8(4), 293-297.

Vukovic, N., Feurra, M., Shpektor, A., Myachykov, A., \& Shtyrov, Y. (2016). Primary motor cortex functionally contributes to language comprehension: an online rTMS study. Neuropsychologia, 96, 222-229.

Vukovic, N., \& Shtyrov, Y. (2014). Cortical motor systems are involved in second-language comprehension: evidence from rapid mu-rhythm desynchronisation. NeuroImage, 102, 695-703.

Vukovic, N., \& Williams, J. N. (2014). Automatic perceptual simulation of first language meanings during second language sentence processing in bilinguals. Acta Psychologica, 145, 98-103.

Vukovic, N., \& Williams, J. N. (2015). Individual differences in spatial cognition influence mental simulation of language. Cognition, 142, 110-122.

Wassenburg, S. I., \& Zwaan, R. A. (2010). Readers routinely represent implied object rotation: the role of visual experience. Quarterly Fournal of Experimental Psychology, 63(9), 1665-1670.

Waters, W. E., \& O'Connor, P. J. (1975). Prevalence of migraine. Fournal of Neurology, Neurosurgery and Psychiatry, 38(6), 613-616.

Yang, J., \& Shu, H. (2016). Involvement of the motor system in comprehension of non-literal action language: a meta-analysis study. Brain Topography, 29(1), 94-107.

Yee, E. (2017). Fluid semantics: semantic knowledge is experience-based and dynamic. In A. Lahiri \& S. Kotzor (Eds.), The speech processing lexicon: neurocognitive and behavioural approaches (pp. 235-254). Berlin: Walter de Gruyter.

Zwaan, R. A., \& Pecher, D. (2012). Revisiting mental simulation in language comprehension: six replication attempts. PloS One, 7(12). Online <https://doi.org/10.1371/journal. pone.0051382>. 